\title{
La FMH opte pour une nouvelle voie
}

\author{
Silvia Cuenia, Rudolf Hauri ${ }^{b}$, Christine Romann ${ }^{c}$ \\ ${ }^{a}$ Dr med., spécialiste en psychiatrie et psychothérapie; ${ }^{b}$ Dr med., spécialiste en médecine légale, médecin cantonal de Zoug;
}

${ }^{c}$ Dr med., membre du Comité central, responsable du département Promotion de la santé et prévention

\begin{abstract}
Dans leur article, «Standesverfahren bei Missbrauch durch Ärzte», paru en juin 2010 [1] dans le BMS, les psychiatres Silvia Cueni et Maya Schuppli-Delpy ont critiqué le comportement des médecins face à leurs confrères faillibles. Ces réflexions ont suscité un débat fructueux au sein de la FMH qui a incité le Comité central à créer un groupe de travail. Après cinq ans de travaux, celui-ci a jeté les bases pour une discussion élargie entre les organes de la FMH, débouchant sur une modification du Code de déontologie.
\end{abstract}

\section{${ }^{*}$ Nous avons opté pour l'usage du féminin, car même si les abus touchent les deux sexes, la grande majorité des abus à caractère sexuel sont perpétrés par des hommes à l'encontre des femmes.}

Le groupe de travail qui a mis fin à son activité souhaite désormais partager et présenter les résultats de ses réflexions tout en soulignant les sujets pour lesquels il est encore nécessaire d'agir.

Pour obtenir une vue d'ensemble de cette thématique difficile et proposer des solutions appropriées, le groupe de travail est parti de l'article 4 du Code de déontologie (CoD) qui fixe des limites claires à l'exercice de la profession médicale: «Dans l'exercice de sa profession, le médecin n'exploite pas un éventuel état de dépendance du patient; il lui est tout particulièrement interdit d'abuser de son autorité sur lui, tant sur le plan émotionnel ou sexuel que matériel.» Très rapidement, il est ressorti que la structure même de la procédure de déontologie posait problème. Jusqu'en 2012, les patientes* pouvaient déposer une dénonciation en première instance auprès des commissions de déontologie cantonales, mais sans possibilité d'être partie à la procédure. Elles ne pouvaient dès lors pas consulter le dossier de la cause ni connaître la version du médecin dénoncé et se trouvaient donc dans l'impossibilité de

Membres du groupe de travail

- P. Ackle, spécialiste en médecine générale

- Ch. Bernath, spécialiste en psychiatrie et psychothérapie

- S. Burkhard Schneider, juriste à I'état-major, ASMAC

- S. Cueni, spécialiste en psychiatrie et psychothérapie

- M. Graf, spécialiste en psychiatrie et psychothérapie, directeur de la clinique

de psychiatrie forensique

- R. Hauri, spécialiste en médecine légale, médecin cantonal de Zoug

- H.-U. Koelz, vice-président de I'ISFM

- G. Lang, avocate, division Service juridique de la FMH

- C. Romann, membre du Comité central de la FMH - direction

- M. Schuppli-Delpy, spécialiste en psychiatrie et psychothérapie

- J. Schlup, président de la FMH

- B. Weil, cheffe de la division Promotion de la santé et prévention de la FMH se déterminer. De plus, elles n'étaient pas informées de l'issue de la procédure ou uniquement de manière très succincte. Pour les patientes ayant subi une violation de la dignité humaine, qui représente un manquement grave de la part du médecin, cette situation était inacceptable. Les exemples de cas discutés au sein du groupe de travail ont par ailleurs clairement montré que les commissions de déontologie ne faisaient que trop rarement usage des sanctions à leur disposition. Si l'on rajoute à cela le manque de transparence de la procédure elle-même, l'effet était particulièrement dissuasif sur les éventuelles dénonciations des patientes. Le groupe de travail a donc rapidement souligné que les patientes devaient obtenir plus de droits dans ces situations délicates et émotionnellement difficiles pour elles. Afin de faciliter le travail des commissions de déontologie, pour lesquelles l'instauration d'une nouvelle réglementation rendait la tâche encore plus ardue, et afin d'unifier la jurisprudence en la matière au plan national, le groupe de travail a estimé que les commissions de déontologie de première instance avaient besoin d'outils non seulement pour échanger entre elles, mais également pour communiquer avec la Commission de déontologie de la FMH compétente en deuxième instance. Il a également jugé que les commissions de déontologie devaient pouvoir consulter les décisions (anonymisées) des autres commissions de déontologie.

Les propositions du groupe de travail ont suscité de vifs débats au sein des organes de la FMH. En avril 2013, la Chambre médicale a finalement décidé que dans les procédures ayant pour objet une atteinte à la dignité humaine ou un abus de l'état de dépendance dans la relation médicale, les patientes devaient obtenir le 
droit de se constituer partie, de consulter les documents relatifs à la procédure et également obtenir d'autres droits. Afin d'épauler les commissions de déontologie dans leurs travaux, il a également été décidé de créer une banque de données nationale recensant toutes les décisions dans ce domaine et d'organiser une première journée d'échange d'expériences pour que les membres des commissions de déontologie cantonales intéressés puissent s'entretenir avec les membres des commissions de déontologie des autres cantons et discuter des difficultés rencontrées lors de la mise en œuvre de la nouvelle réglementation.

Mais il importe aussi de prévenir toute violation de l'article 4 du Code de déontologie de la FMH et de rendre les membres de la FMH attentifs aux conséquences de leurs actes. A la demande du groupe de travail, l'Institut suisse pour la formation médicale postgraduée et continue (ISFM) a intégré le paragraphe suivant dans son catalogue des objectifs généraux de formation: «Le spécialiste est conscient de la situation de dépendance de ses patientes et patients et n'abuse pas de son pouvoir pour imposer ses propres intérêts. Il n'accepte aucun cadeau inadéquat, aucun traitement de faveur et aucun avantage, il renonce à tout endoctrinement idéologique ou religieux, et en particulier, il s'abstient de toute forme de contact sexuel.» Un jalon a ainsi été posé pour sensibiliser les futurs spécialistes à la question des limites à respecter dans la relation avec les patientes et aux conséquences d'une violation de ces limites tant pour la patiente que pour eux-mêmes. L'application concrète de cette mesure dans la formation postgraduée et continue doit encore être développée avec le soutien des sociétés de discipline médicale. La Société suisse de psychiatrie et psychothérapie s'est pour sa part prononcée de manière décisive dans un papier de position et la Société suisse de gynécologie et obstétrique a édicté des directives exemplaires montrant clairement les limites à respecter dans le contact avec les patientes. Et en dernier recours, si les mesures de prévention devaient rester sans effets, le médecin concerné pourra être adressé à ReMed, le réseau de soutien pour les médecins en situation de crise.

Les patientes concernées peuvent également s'adresser directement aux autorités cantonales de surveillance, mais là aussi, le groupe de travail a identifié le besoin d'agir. Les expériences faites en psychothérapie avec des patientes victimes d'abus sexuels par des médecins ont en effet montré que les autorités compétentes réagissaient très différemment d'un canton à l'autre. Les commissions de déontologie peuvent notamment prononcer une sanction en notifiant un cas à l'autorité cantonale de surveillance qui joue un rôle décisif dans l'octroi des autorisations de pratiquer. En vertu de la loi sur les professions médicales, il incombe aux autorités cantonales de surveillance de déterminer si un médecin qui a failli dispose des compétences humaines et médicales nécessaires pour (continuer à) exercer sa profession, ce qui inclut aussi expressément de déterminer s'il est digne de confiance. Si cette dernière condition n'est pas remplie, alors le médecin se verra retirer son autorisation de pratiquer pour une durée indéterminée. Les autorités de surveillance doivent également évaluer si des mesures disciplinaires, telles que mise en garde, blâme ou amende, ou si un retrait temporaire de l'autorisation de pratiquer, des restrictions ou des conditions particulières doivent être prononcés. A long terme, de telles mesures peuvent restreindre considérablement la liberté économique des médecins concernés. Une position claire de la FMH constitue un soutien essentiel pour les autorités de surveillance dans l'accomplissement de leurs tâches. Il faut toutefois, à titre d'exemple, pouvoir identifier clairement les actes qui sont considérés comme une violation de l'art. 4 du Code de déontologie.

\section{La Société suisse de gynécologie et obstétrique a édicté des directives exemplaires montrant clairement les limites à respecter dans le contact avec les patientes.}

Afin de développer une compréhension commune et de sensibiliser et soutenir au besoin les médecins cantonaux dans ce domaine, le groupe de travail a présenté ses travaux à l'Association des médecins cantonaux de Suisse (AMCS) qui a fait preuve d'ouverture. La FMH prévoit donc de poursuivre son travail de sensibilisation, en collaboration avec l'AMCS, pour interpeller et sensibiliser la Conférence des directeurs cantonaux de la santé (CDS) à ces questions. De par ses décisions, le corps médical a souligné qu'il saluait une autorité de surveillance forte et qu'il était prêt à s'engager pour une collaboration efficiente.

Davantage de droits pour les patientes dans les procédures de déontologie, une position claire de l'ISFM, le soutien des commissions de déontologie cantonales et la collaboration avec l'AMCS ont été les premières étapes du travail accompli à ce jour. Poursuivre la sensibilisation des futurs médecins spécialistes et approfondir la réflexion autour de ces questions tout au long de la vie professionnelle sont des sujets sur lesquels le corps médical doit encore se pencher.

\footnotetext{
Références

1 Cueni S, Schuppli-Delpy M. Standesverfahren bei Missbrauch durch Ärzte. Bull Méd Suisses; 2010;91(16):645-7.
} 